\title{
Absolute phase birefringence dispersion in polarization-maintaining fiber or birefringent crystal retrieved from channeled spectrum
}

\author{
Petr Hlubina, ${ }^{*}$ Dalibor Ciprian \\ Department of Physics, Technical University Ostrava, \\ 17. listopadu 15, 70833 Ostrava-Poruba, Czech Republic \\ *Corresponding author: petr.hlubina@vsb.cz
}

Compiled March 22, 2010

\begin{abstract}
We report on a simple method for retrieving the wavelength dependence of the phase birefringence in a polarization-maintaining fiber or a birefringent crystal from a channeled spectrum. The method utilizes interference of polarized modes or waves resolved as the channeled spectrum and its processing by a windowed Fourier transform to reconstruct precisely the phase as a function of wavelength. The ambiguity of the phase is removed provided that we know both the approximative function for the birefringence dispersion and the length of the fiber or the thickness of the crystal. The method is used in measuring the wavelength dependence of the phase birefringence in an elliptical-core fiber or in a quartz crystal in a range from 500 to $900 \mathrm{~nm}$. The dependences are compared with those resulting from the available data, and very good agreement is confirmed. (C) 2010 Optical Society of America

OCIS codes: $060.2300,060.2420,120.3180,260.1180,260.1440,260.2030$
\end{abstract}

Highly-birefringent, polarization-maintaining fibers (PMFs) have attracted considerable interest for a number of applications, including e.g. polarizationsensitive optical devices and fiber-optic sensors of various physical quantities employing interferometric techniques. For these applications, it is important to know the birefringence dispersion, i.e., the wavelength dependence of the phase and group birefringence in the PMFs. Several methods have been developed to measure the phase birefringence in PMFs over a wide spectral range. A wavelength scanning technique can be applied to either short [1] or long [2] fibers.

It is well known that scanning the wavelength alone (a channeled spectrum) gives only a relative measure of the phase birefringence and it inherently measures the group birefringence. The absolute phase birefringence is obtained by both scanning the wavelength and absolutely measuring the birefringence at one particular wavelength. To measure this quantity, a precision electromagnetic modulation technique [1] or a lateral force method [3] applied in the time [4] or wavelength [5] domain can be used. Similarly, the birefringence dispersion is of fundamental importance for anisotropic materials used in optical devices such as wave plates, compensators, retarders and polarizers. Measurement of the phase birefringence of a prescribed dispersion function can be performed by spectral interferometric techniques based on either determining the positions of maxima in a channeled spectrum [6] or by fitting the measured spectrum to the theoretical one [7].

In this Letter, a simple method for retrieving the wavelength dependence of the phase birefringence in a PMF of known length is presented. The technique, which is based on processing of a channeled spectrum to retrieve the phase function, utilizes the approximative function of the phase birefringence dispersion. We extended the use of the technique for measuring the phase birefringence in a quartz crystal of known thickness.

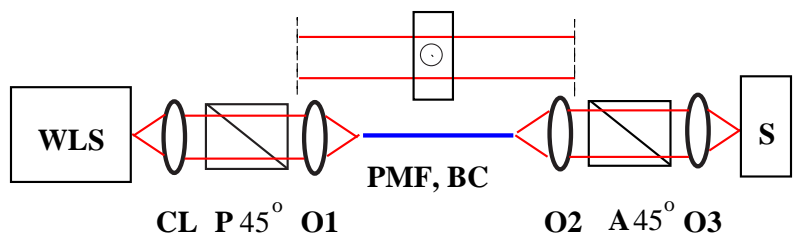

Fig. 1. (Color online) Experimental setup to record a channeled spectrum for a PMF or a birefringent crystal.

Consider a PMF supporting two polarization modes over a broad spectral range. We can introduce the wavelength-dependent phase birefringence $B(\lambda)=$ $n_{x}(\lambda)-n_{y}(\lambda)$, where $n_{x}(\lambda)$ and $n_{y}(\lambda)$ are the effective phase refractive indices for the respective polarization modes. Figure 1 illustrates a simple experimental setup we use for recording of a channeled spectrum from which we retrieve the wavelength dependence of $B(\lambda)$ in a PMF, if its length $L$ is known precisely. Light from white-light source WLS passes through collimator CL and polarizer $\mathrm{P}$, and is launched by microscope objective O1 into the PMF under test. The transmission azimuth of the polarizer is adjusted at $45^{\circ}$ with respect to the polarization axes of the PMF so both polarization modes are excited equally in the tested fiber. The two polarization modes are mixed with analyzer $\mathrm{A}$, which is placed between microscope objectives $\mathrm{O} 2$ and $\mathrm{O} 3$, and their interference is resolved by spectrometer $\mathrm{S}$ as a channeled spectrum. The transmission azimuth of the analyzer is adjusted at $45^{\circ}$ with respect to the polarization axes of the PMF. The spectral intensity recoded by the spectrometer of a Gaussian response function can be represented in the form [8]

$$
I(\lambda)=I_{0}(\lambda)\left\{1+V_{\mathrm{R}}(\lambda) \cos [(2 \pi / \lambda) B(\lambda) L]\right\},
$$


where $I_{0}(\lambda)$ is the reference (unmodulated) spectrum and $V_{\mathrm{R}}(\lambda)=\exp \left\{-\left(\pi^{2} / 2\right)\left[G(\lambda) L \Delta \lambda_{\mathrm{R}} / \lambda^{2}\right]^{2}\right\}$ is a visibility term dependent on both the group birefringence $G(\lambda)=-\lambda^{2} \mathrm{~d}[B(\lambda) / \lambda] / \mathrm{d} \lambda$ and the width $\Delta \lambda_{\mathrm{R}}$ of the spectrometer response function. To resolve a channeled spectrum (spectral fringes) in a spectral range from $\lambda_{1}$ to $\lambda_{2}$, the fiber length must satisfy the condition $L<\lambda_{1}^{2} /\left[G\left(\lambda_{1}\right) \Delta \lambda_{\mathrm{R}}\right]$.

From the recorded channeled spectrum, a relative spectral phase $\Phi_{r}(\lambda)$ can be retrieved with the ambiguity of $m 2 \pi$, where $m$ is an integer. To remove the phase ambiguity, we assume that the phase birefringence dispersion is given by the relation [9]

$$
B(\lambda)=A_{1} \lambda^{-4}+A_{2} \lambda^{-2}+A_{3}+A_{4} \lambda^{2}+A_{5} \lambda^{4},
$$

where $A_{i}$ are the coefficients. The difference between the retrieved phase function and the absolute phase function is the phase error function

$$
e(\lambda)=\Phi_{r}(\lambda)+m 2 \pi-(2 \pi / \lambda) B(\lambda) L,
$$

which is a measure of the correct determination of the coefficients $A_{i}$ and the interference order $m$. In an ideal case $e(\lambda)=0$.

The experimental setup used for measurement of the phase birefringence $B(\lambda)$ in a PMF is shown schematically in Fig. 1 and it consists of a halogen lamp HL2000 (Ocean Optics), a collimating lens, microscope objectives $(10 \times / 0.30)$, Glan Taylor calcite polarizer and analyzer (Thorlabs), an elliptical-core PMF of length $L=(356.5 \pm 0.5) \mathrm{mm}$, a fiber-optic spectrometer S2000 (Ocean Optics) and other components. The PMF is with the core made of $\mathrm{GeO}_{2}$-doped silica glass (19.3 mol \%) and the cladding made of pure silica. The dimensions of the fibre elliptical core are approximately $3.2 \mu \mathrm{m} \times 1.2$ $\mu \mathrm{m}$. The spectrometer with $\Delta \lambda_{\mathrm{R}} \approx 3 \mathrm{~nm}$ has a spectral operation range from 350 to $1000 \mathrm{~nm}$.

We demonstrate the ability of our method in measuring the phase birefringence $B(\lambda)$ in the PMF for which the channeled spectrum shown in Fig. 2 was recorded. It is clearly seen that a large number of the spectral interference fringes of sufficiently high visibility are resolved. Procedure used to retrieve a relative spectral phase $\Phi_{r}(\lambda)$ from the recorded channeled spectrum comprises two steps. In the first step, the reference spectrum $I_{0}(\lambda)$ is retrieved from the channeled spectrum $I(\lambda)$. It is obtained as the inverse Fourier transform of the zero-order component of the Fourier spectrum of the recorded channeled spectrum. In the second step, the spectral phase function $\Phi_{r}(\lambda)$ is retrieved from the spectral signal $S(\lambda)=I(\lambda) / I_{0}(\lambda)-1$ using a procedure based on a windowed Fourier transform [10].

The procedure used in the determining the coefficients $A_{i}$ in Eq. (2) consists of two steps. In the first step, a trust-region algorithm [11] is used for the retrieved $\Phi_{r}(\lambda)$ to estimate $m$ and $A_{i}$ that give the absolute phase function. The integer value of $m$ is used in the second step when the coefficients $A_{i}$ are obtained by using the

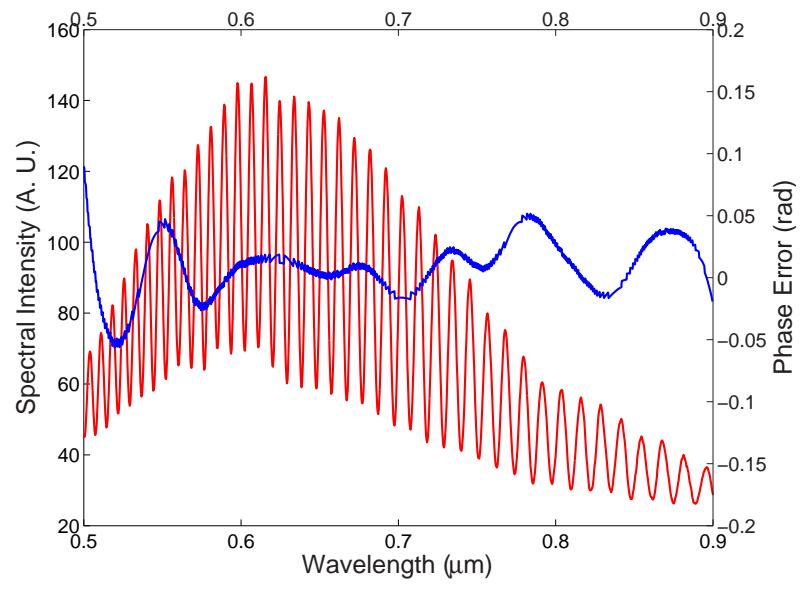

Fig. 2. (Color online) Channeled spectrum recorded for a PMF and the phase error function $e(\lambda)$ obtained by our procedure.

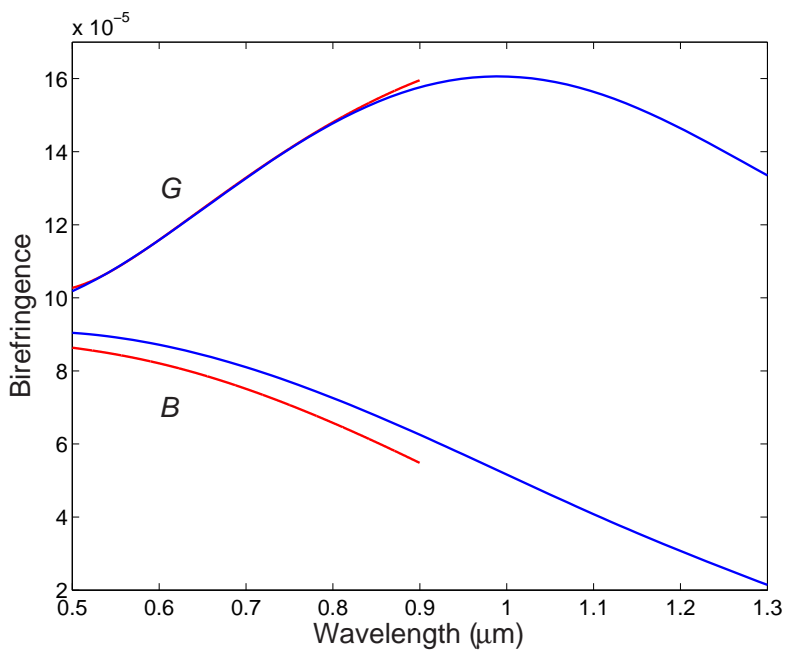

Fig. 3. (Color online) Retrieved phase and group birefringences, $B(\lambda)$ and $G(\lambda)$, for a PMF compared with those measured over a broader spectral range.

Levenberg-Marquardt least-squares algorithm that minimizes the function $\chi^{2}=\sum_{i=1}^{N} e^{2}\left(\lambda_{i}\right)$, where $\lambda_{i}$ are wavelengths at which the spectrum was recorded. The procedure gives the phase error function $e(\lambda)$ shown in Fig. 2. Note that this function consists of the artifacts (due to the phase retrieval procedure [10]) that oscillate around the zero value. Using the coefficients $A_{i}$ and Eq. (2), the absolute phase birefringence $B(\lambda)$ shown in Fig. 3 was determined. For an error of $\pm 0.5 \mathrm{~mm}$ in the length of the $\mathrm{PMF}$ a relative error in the birefringence is about $0.1 \%$. Contribution of the phase error $e(\lambda)= \pm 0.1 \mathrm{rad}$ to the relative error is even smaller. In Fig. 3 is also shown the birefringence $B(\lambda)$ measured over a broader wavelength range. It was retrieved from the group birefringence $G(\lambda)$ measured by a technique of tandem interferometry [12] and using $B\left(\lambda_{0}\right)=8.55 \times 10^{-5}$ at $\lambda_{0}=637.08 \mathrm{~nm}$ measured by a lateral force method applied in the wavelength 
domain [5]. There is a nearly constant shift between these dependences and our method gives $B\left(\lambda_{0}\right)=7.32 \times 10^{-5}$ what represents $14 \%$ difference between the measurement results. The discrepancy is probably caused by different birefringences of fiber samples used in the experiments. Figure 3 demonstrates very good agreement between the group birefringences $G(\lambda)$ measured by both techniques.

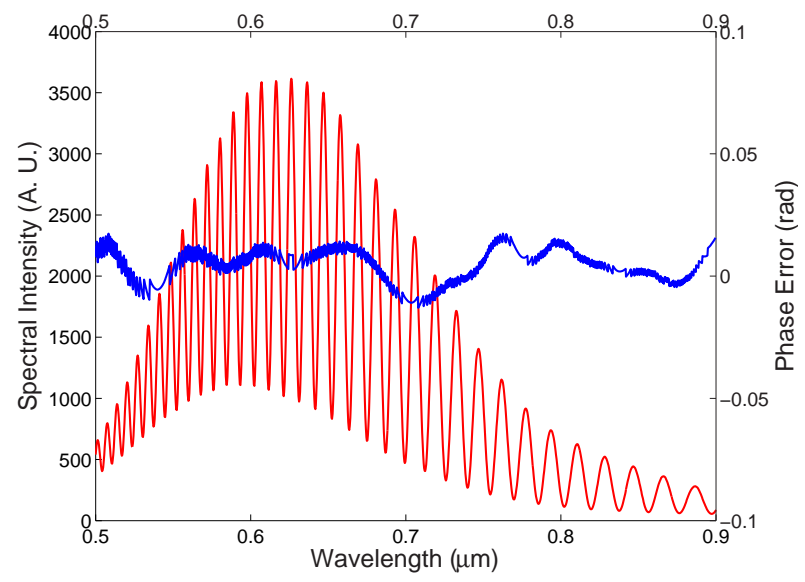

Fig. 4. (Color online) Channeled spectrum recorded for a birefringent quartz and the phase error function $e(\lambda)$ obtained by our procedure.

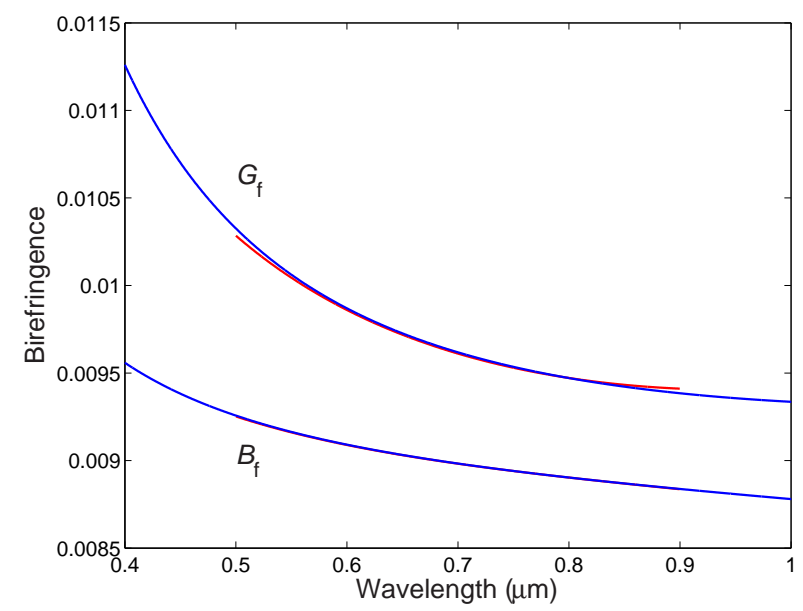

Fig. 5. (Color online) Retrieved phase and group birefringences, $B_{\mathrm{f}}(\lambda)$ and $G_{\mathrm{f}}(\lambda)$, for a birefringent quartz compared with those given by the dispersion relation.

We extended the use of our method in determining the phase birefringence $B_{\mathrm{f}}(\lambda)=n_{e}(\lambda)-n_{o}(\lambda)$ in a quartz crystal, where $n_{e}(\lambda)$ and $n_{o}(\lambda)$ are the phase refractive indices of the extraordinary and ordinary eigenwaves, respectively. Our setup was modified (see the upper part of Fig. 1) and for the crystal of thickness $t=(4010 \pm 1) \mu \mathrm{m}$ and the orientation of the optic axis shown in Fig. 1 we recorded the channeled spectrum shown in Fig. 4. It is clearly seen that a large number of the spectral interference fringes of sufficiently high vis- ibility are resolved. The number of the spectral fringes in the wavelength range from 500 to $900 \mathrm{~nm}$ is given by the group path difference $G_{\mathrm{f}}(\lambda) t$ in the crystal. Using the same procedure as presented above, we retrieved the absolute birefringence $B_{\mathrm{f}}(\lambda)$ shown in Fig. 5. For the phase error $e(\lambda) \approx 0.03 \mathrm{rad}$ a relative error in the birefringence is about $0.03 \%$. Contribution of the thickness uncertainty to the relative error is even smaller. The same figure shows the birefringence $B_{\mathrm{f}}(\lambda)$ resulting from the Sellmeier-like form of the dispersion relation for the quartz crystal [8]. We clearly see that there is very good agreement between these dependences that have slightly different dispersion slopes. This is illustrated in Fig. 5 that shows the dispersion functions for the group birefringence $G_{\mathrm{f}}(\lambda)=-\lambda^{2} \mathrm{~d}\left[B_{\mathrm{f}}(\lambda) / \lambda\right] / \mathrm{d} \lambda$. The difference between them, which is in general dependent on the fitting function, is in part caused by the approximation (2) of the phase birefringence dispersion $B_{\mathrm{f}}(\lambda)$ we used.

In conclusion, a simple technique for retrieving the absolute phase birefringence from a channeled spectrum has been presented. It utilizes the approximative function of the birefringence dispersion and the phase retrieval using a windowed Fourier transform. The feasibility of the technique has been demonstrated in measuring the phase birefringence in a PMF or in a quartz crystal. We confirmed very good agreement with the available data. The use of the technique can be extended, e.g., for retrieving the dispersion of the differential phase refractive index from a channeled spectrum originated from interference of two spatial modes guided in a fiber.

The research has been partially supported by the Ministry of Education, Youth and Sports of the Czech Republic through grant MSM6198910016, and by the regional grant CZ.1.05/2.1.00/01.0040.

\section{References}

1. S. C. Rashleigh, Opt. Lett. 7, 294 (1982).

2. M. G. Shlyagin, A. V. Khomenko, and D. Tentori, Opt. Lett. 20, 869 (1995).

3. K. Takada, J. Noda, and R. Ulrich, Appl. Opt. 24, 4387 (1985).

4. W. J. Bock and W. Urbanczyk, Appl. Opt. 32, 5841 (1993).

5. P. Hlubina and D. Ciprian, Opt. Express 15, 17019 (2007).

6. M. Medhat and S. Y. El-Zaiat, Opt. Commun. 141, 145 (1997).

7. H. Delbarre, M. Przygodzki, C. Tassou, and D. Boucher, Appl. Phys. B 70, 45 (2000).

8. P. Hlubina, D. Ciprian, and L. Knyblova, Opt. Commun. 260, 535 (2006).

9. M. Tsubokawa, N. Shibata, T. Higashi, and S. Seikai, J. Opt. Soc. Am. A 4, 1895 (1987).

10. P. Hlubina, J. Lunacek, D. Ciprian, and R. Chlebus, Opt. Commun. 281, 2349 (2008).

11. Curve Fitting Toolbox for Use with MATLAB (MathWorks, 2000).

12. P. Hlubina, D. Ciprian, and M. Kadulova, Meas. Sci. Technol. 20, 025301 (2009). 